\title{
ALS Superbend Magnet Performance
}

\author{
S. Marks, J. Zbasnik, W. Byrne, D. Calais, M. Chin, R. DeMarco, M. Fahmie, A. Geyer, J. Krupnick, \\ F. Ottens, J. A. Paterson, P. Pipersky, D. A. Robin, R. D. Schlueter, C. Steier, A. Wandesforde
}

\begin{abstract}
${ }^{1}$ Abstract--The Lawrence Berkeley National Laboratory has been engaged in the design, construction and testing of four superconducting dipoles (Superbends) that are installed in three arcs of the Advanced Light Source (ALS), with the fourth magnet as a spare. This represents a major upgrade to the ALS providing an enhanced flux and brightness at photon energies above $10 \mathrm{keV}$. In preparation for installation, an extensive set of tests and measurements have been conducted to characterize the magnetic and cryogenic performance of the Superbends and to fiducialize them for accurate placement in the ALS storage ring. The magnets are currently installed, and the storage ring is undergoing final commissioning. This paper will present the results of magnetic and cryogenic testing.
\end{abstract}

\section{INTRODUCTION}

$\mathrm{T}$ he Advanced Light Source (ALS) at the Lawrence Berkeley National Laboratory (LBNL) is a national user facility producing high brightness synchrotron radiation, primarily in the vacuum ultraviolet and soft x-ray regimes (6 $\mathrm{eV}$ to $10 \mathrm{keV}$ ). The ALS has engaged in a major upgrade to meet the growing demand for bright hard x-ray sources with photon energies above $10 \mathrm{keV}$. The upgrade consists of replacing three of the total of 36 conventional storage ring bending magnets with three superconducting bend magnets (Superbends). The increase in peak field from $1.34 \mathrm{~T}$, corresponding to the conventional bend magnets, to $5.74 \mathrm{~T}$, for the Superbends, results in almost an order of magnitude increase in both flux and brightness at $10 \mathrm{keV}$, and nearly two orders of magnitude at $20 \mathrm{keV}$ for an electron beam energy of $1.9 \mathrm{GeV}$. Experimental uses for the new sources will include protein crystallography, x-ray tomography and powder diffraction.

In the initial R\&D phase of the Superbend project a conceptual design of a magnet that could be incorporated into the ALS was developed, and a robust superconducting coil was produced and tested[1], [2]. The second phase of the project, a joint venture between LBNL and Wang NMR, involved detailed design and fabrication of four magnets.

Following fabrication, each magnet underwent extensive magnetic and cryogenic testing. This paper will report the results of the tests. Section II will summarize magnetic test

Manuscript received September 24, 2001. This work was supported by the Director, Office of Energy Research, Office of Basic Energy Sciences, Materials Sciences Division, of the U. S. Department of Energy, under Contract No. DE-AC03-76F00098.

Authors are with Larwence Berkeley National Laboratory, 1 Cyclotron Rd., Berkeley, California, USA. results; Section III will summarize cryogenic test results. The design, requirements, and Superbend project have been discussed elsewhere [3], [4], [5].

\section{MAGNETIC PERFORMANCE}

\section{A. Magnetic Measurement Setup}

Figure 1 below, shows a picture of Superbend \#1 installed around an alignment fixture on the test stand. Both the cryostat and the alignment fixture are mounted with six struts allowing for adjustment in all degrees of freedom.

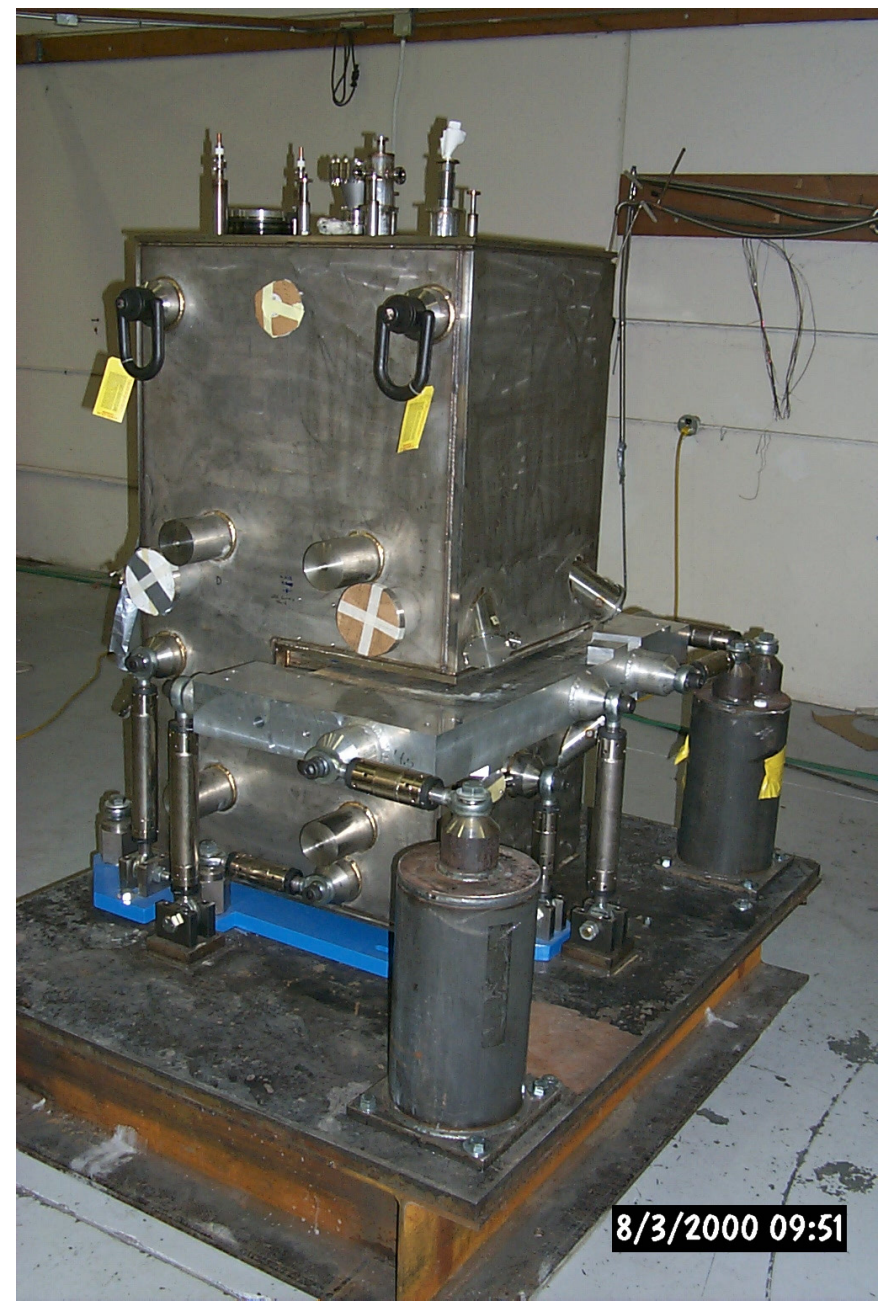

Fig. 1 Magnet \#1 installed on the test stand.

The alignment fixture serves several important functions in the testing process [4]. A cylindrical bore coincident with the 
electron beam axis is drilled through the alignment fixture. The bore is used to position measurement coils and probes. As explained in the Section $B$ below, multipole measurements are used to position the magnet and cryostat so that the magnetic axis coincides with the measurement, or alignment fixture bore, axis. Fiducials on the alignment fixture are used to relate this axis to fiducials on the cryostat, which are then used as survey marks for installation relative to the storage ring vacuum chamber. The alignment fixture is machined with the same profile as the Superbend cutout in the storage ring vacuum chamber, thus insuring correct fit during installation.

The primary operating energy range for the ALS is between $1.5 \mathrm{GeV}$ and $1.9 \mathrm{GeV}$. The corresponding Superbend current range is approximately 212 A to 298 A. Magnetic measurements were conducted in the range between $200 \mathrm{~A}$ and $300 \mathrm{~A}$.

\section{B. Multipoles}

Multipoles are measured with a $1 \mathrm{~m}$ long integral search coil [4]. Multipole measurements are made to insure that field quality tolerances are met and to locate the magnetic axis.

\section{1) Requirements}

Table I below is a summary of normalized Superbend multipole requirements conforming to the following multipole definition

$$
\bar{B}^{*}=\bar{B}_{x}-i \bar{B}_{y}=i \sum_{n}\left(\bar{a}_{n}+i \bar{b}_{n}\right)\left(\frac{z}{r_{0}}\right)^{n-1}[1] \text {. }
$$

The symbol indicates integral quantities. Multipoles are evaluated at the normalization radius, $r_{0}=1 \mathrm{~cm}$.

TABLE I

SUPERBEND MULTIPOLE REQUIREMENTS AT 1 CM RADIUS

\begin{tabular}{cccc}
\hline \hline $\boldsymbol{n}$ & $\left|\overline{\boldsymbol{a}}_{\boldsymbol{n}} / \overline{\boldsymbol{a}}_{\mathbf{1}}\right|$ & $\left|\Delta \overline{\boldsymbol{a}}_{\boldsymbol{n}} / \overline{\boldsymbol{a}}_{\mathbf{1}}\right|$ & $\left|\overline{\boldsymbol{b}}_{\boldsymbol{n}} / \overline{\boldsymbol{a}}_{\mathbf{1}}\right|$ \\
\hline 1 & 1 & $1 \times 10^{-3}$ & $2.5 \times 10^{-4}$ \\
2 & --- & $7.6 \times 10^{-4}$ & $4.7 \times 10^{-4}$ \\
3 & $6 \times 10^{-3}$ & $3 \times 10^{-4}$ & $2 \times 10^{-4}$ \\
$n>3$ & $2 \times 10^{-4}$ & --- & $2 \times 10^{-4}$ \\
\hline \hline
\end{tabular}

The $\Delta$ symbol indicates a requirement on magnet-tomagnet reproducibility. The requirement on $\bar{b}_{1}$ corresponds to the $0.25 \mathrm{mrad}$ magnet roll requirement, which will be discussed in Section $C$. The $\Delta \bar{a}_{1}$ value corresponds to a requirement on power supply tracking during magnet ramping. The $\Delta \bar{a}_{2}$ requirement is based upon the horizontal orbit and vertical $\beta$-beat tolerances; $\bar{b}_{2}$ is based upon vertical orbit distortion and coupling tolerances. The $\bar{a}_{3}$ and $\Delta \bar{a}_{3}$ requirements are based upon electron orbit simulations.

\section{2) Sextupole and higher order multipoles}

The measured value for $\bar{a}_{3} / \bar{a}_{1}$ varied between $3.20 \times 10^{-3}$ and $3.26 \times 10^{-3}$ for the four magnets, easily meeting the specifications. The sextupole value agrees very well with the value $3.23 \times 10^{-3}$, determined from three-dimensional finite element models using the Tosca code. All higher order multipoles were less than $1 \times 10^{-4}$.

\section{3) Transverse and Vertical Position}

The transverse and vertical magnetic center is defined as the center of the sextupole, the dominant multipole component. In the absence of other asymmetries giving rise to a quadrupole, this is the location where the quadrupole component is zero. Consider the sextupole term with an offset of the magnetic center by an amount $\Delta z$.

$$
\bar{B}_{3}^{*}=\bar{c}_{3}\left(\frac{z+\Delta z}{r_{0}}\right)^{2}=\bar{c}_{3}\left(\frac{z^{2}+2 \Delta z z+\Delta z^{2}}{r_{0}^{2}}\right)
$$

The apparent quadrupole component is

$$
\bar{c}_{2}=2 \frac{\Delta z}{r_{0}} \bar{c}_{3} \text {. }
$$

The magnetic center offset is determined as

$$
\Delta z=\Delta x+i \Delta y=\frac{1}{2} r_{0} \frac{\overline{c_{2}}}{\overline{c_{3}}} .
$$

This procedure was used as the first step in the magnetic measurement process for each of the four Superbend magnets. Setting the magnet current at $250 \mathrm{~A}$, roughly in the middle of the normal operating range, measured values of $\overline{c_{2}}$ and $\bar{c}_{3}$ were used to position the magnet assemblies in order to cancel the quadrupole components. This method worked very well, as is illustrated in Table II, which shows the results of magnet \#3 positioning. Table III shows the quadrupole values for all four magnets after the final adjustment.

TABLE II

TRANSVERSE AND VERTICAL CENTER ADJUSTMENT FOR MAGNET \#3

\begin{tabular}{lcccc}
\hline \hline & $\overline{\boldsymbol{a}}_{\mathbf{2}} / \overline{\boldsymbol{a}}_{\mathbf{1}}$ & $\begin{array}{c}\Delta \boldsymbol{x} \\
{[\mathrm{mm}]}\end{array}$ & $\overline{\boldsymbol{b}}_{\mathbf{2}} / \overline{\boldsymbol{a}}_{\mathbf{1}}$ & $\begin{array}{c}\Delta \boldsymbol{y} \\
{[\mathrm{mm}]}\end{array}$ \\
\hline $\begin{array}{l}\text { Initial } \\
\text { Position }\end{array}$ & $4.84 \times 10^{-4}$ & -0.75 & $3.20 \times 10^{-5}$ & -0.07 \\
$\begin{array}{l}\text { After Moving } \\
\Delta \mathrm{x}=0.76 \mathrm{~mm}\end{array}$ & $-3 \times 10^{-6}$ & 0.01 & $4 \times 10^{-4}$ & -0.07 \\
\hline \hline
\end{tabular}

TABLE III

MEASUREd QuAdrupole VAlues AFTER FinAL Positioning

\begin{tabular}{cccc}
\hline \hline Magnet \# & I [A] & $\overline{\boldsymbol{a}}_{\mathbf{2}} / \overline{\boldsymbol{a}}_{\mathbf{1}}$ & $\overline{\boldsymbol{b}}_{\mathbf{2}} / \overline{\boldsymbol{a}}_{\mathbf{1}}$ \\
\hline 1 & 200 & $*$ & $*$ \\
1 & 250 & $*$ & $*$ \\
1 & 300 & $-0.4 \times 10^{-4}$ & $-1.4 \times 10^{-4}$ \\
2 & 200 & $0.9 \times 10^{-4}$ & $0.09 \times 10^{-4}$ \\
2 & 250 & $-0.3 \times 10^{-4}$ & $0.3 \times 10^{-4}$ \\
2 & 300 & $-2.1 \times 10^{-4}$ & $-0.5 \times 10^{-4}$ \\
3 & 200 & $1.5 \times 10^{-4}$ & $0.6 \times 10^{-4}$ \\
3 & 250 & $0.06 \times 10^{-4}$ & $0.4 \times 10^{-4}$ \\
3 & 300 & $-1.4 \times 10^{-4}$ & $-0.4 \times 10^{-4}$ \\
4 & 200 & $1.0 \times 10^{-4}$ & $0.2 \times 10^{-4}$ \\
4 & 250 & $-0.04 \times 10^{-4}$ & $0.7 \times 10^{-4}$ \\
4 & 300 & $-2.0 \times 10^{-4}$ & $-0.2 \times 10^{-4}$ \\
\hline \hline
\end{tabular}


*An adjustment in the magnet assembly was made at the end of measurement for unit \#1. Due to schedule pressures, final measurements were made only at $300 \mathrm{~A}$.

Notice that the normal quadrupole component, $\bar{a}_{2}$, shifts systematically with current. This is caused by the opening and closing of the magnet yoke, thus changing pole angles, due to magnetic forces. However, all measured values are well within the specified tolerance.

\section{Magnet Roll}

The magnet roll tolerance of $0.25 \mathrm{mrad}$ is based on the allowable vertical orbit distortion during normal operation of the storage ring. However, electron beam dynamics studies suggests that as much as $2 \mathrm{mrad}$ of roll can be tolerated for electron beam injection and storage. During the design phase, the difficulty of measuring and adjusting the roll to the required precision was recognized. Therefore, a set of trim coils was designed to produce a skew dipole field, allowing for roll adjustment of several mrad. This reduces the requirement on measurement precision to about $1 \mathrm{mrad}$ to insure that the uncorrected roll is within the $2 \mathrm{mrad}$ required for injection. The trim coils can then be used for fine tuning. Figure 2 shows the trim coils mounted to the cryostat wall.

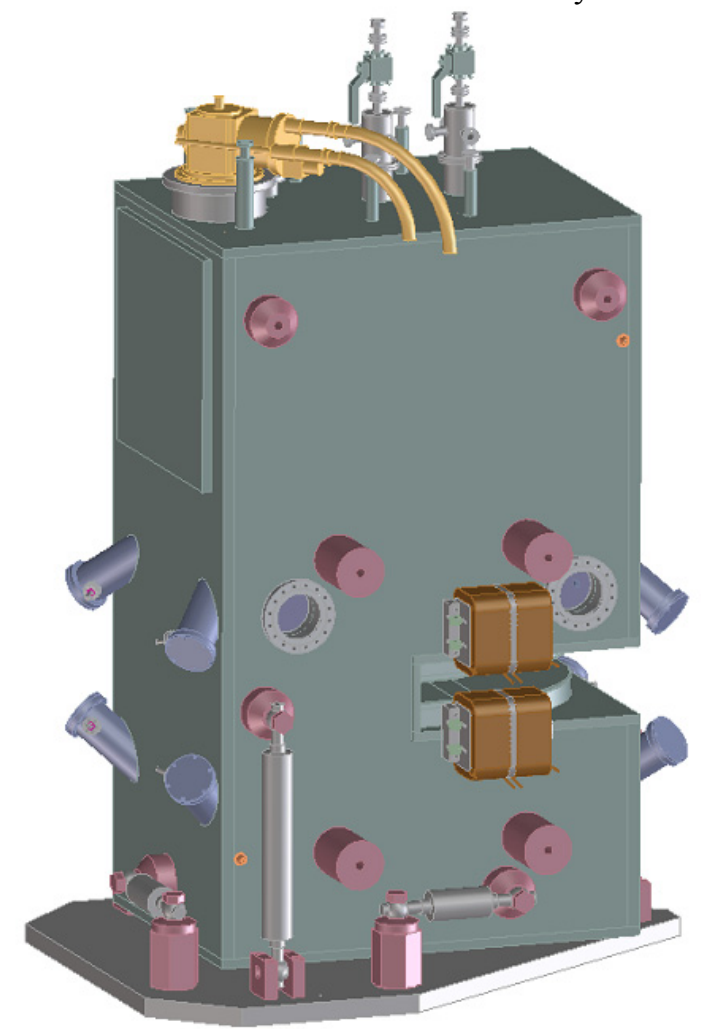

Fig. 2 Trim coils mounted to cryostat wall.

An apparatus incorporating a Hall probe and a precision tilt sensor, with $5 \mu \mathrm{rad}$ resolution, was used for measuring angular field orientation. The Hall probe and tilt sensor holder is attached via a connection rod to a rotating stage, allowing measurement scans of field vs. angle. The rod is inserted into the same alignment fixture bore used to locate the integral search coil, thus insuring roll measurements are done about the magnetic axis. The connection is designed so that the Hall probe and tilt sensor can be inserted from either end of the alignment fixture.

This setup allowed for the detection of field zero as well as field peak. The field zero detection method proved to be unrepeatable due to a strong planar field in the Hall probe at the zero field orientation [2]. The field peak detection method, however, proved to be very repeatable.

A scan consists of 40 data points over a range of $\pm 0.20 \mathrm{rad}$. The data is fit to a function of the form $a \cos \theta+b \sin \theta$. The angular field center, $\theta_{0}$ can then be determined.

$$
\cos \left(\theta+\theta_{0}\right)=a \cos \theta+b \sin \theta ; \theta_{0}=-\tan ^{-1}\left(\frac{b}{a}\right)
$$

Scans are done at the axial location of the peak field and at $\pm 50 \mathrm{~mm}$ and $\pm 100 \mathrm{~mm}$. At each location, five scans are conducted to check for repeatability. The entire scan series is repeated twice, with the Hall probe and tilt sensor apparatus installed into the alignment fixture from both directions. This allows for the subtraction of the fixed angular offset of the tilt sensor relative to the Hall probe.

Table IV is a summary of effective roll for the four Superbend magnets at the two operating currents, $212 \mathrm{~A}$ and $300 \mathrm{~A}$. Effective roll is defined as a weighted average of the roll values measured at the different axial locations, where the weighting factors are based upon the axial field distribution.

TABLE IV

EFFECTIVE ROLL

\begin{tabular}{ccc}
\hline \hline Magnet \# & $\begin{array}{c}\text { Roll @ 212 A } \\
\text { [mrad] }\end{array}$ & $\begin{array}{c}\text { Roll @ 300 A } \\
\text { [mrad] }\end{array}$ \\
\hline 1 & 1.1 & 1.1 \\
2 & 0.0 & 0.0 \\
3 & 0.6 & 0.6 \\
4 & 0.7 & 0.6 \\
\hline \hline
\end{tabular}

\section{Axial Field Profile}

Axial field profiles were used to measure the axial magnetic center and field integrals. They were measured with a Hall probe attached to the end of the integral coil housing. A linear stage incorporated into the integral coil setup allows field scanning along the same axis as is used for the multipole and roll measurements. Figure 3 shows a plot of axial field profiles at $212 \mathrm{~A}$ and $300 \mathrm{~A}$ for magnet \#2.

The axial magnetic center is defined as the location, $z_{0}$, for which the downstream field integral equals the upstream field integral.

$$
\int_{-\infty}^{z_{0}} B_{y} d z=\int_{z_{0}}^{\infty} B_{y} d z
$$

For a symmetric distribution, this also corresponds to the peak field location. The magnetic center must be determined and the magnet placed into the ring accordingly to within 1 $\mathrm{mm}$ in order to meet the tolerance for horizontal orbit distortion. The measured difference between the peak field location and the half-integral point is less than $0.1 \mathrm{~mm}$ for all four magnets. 


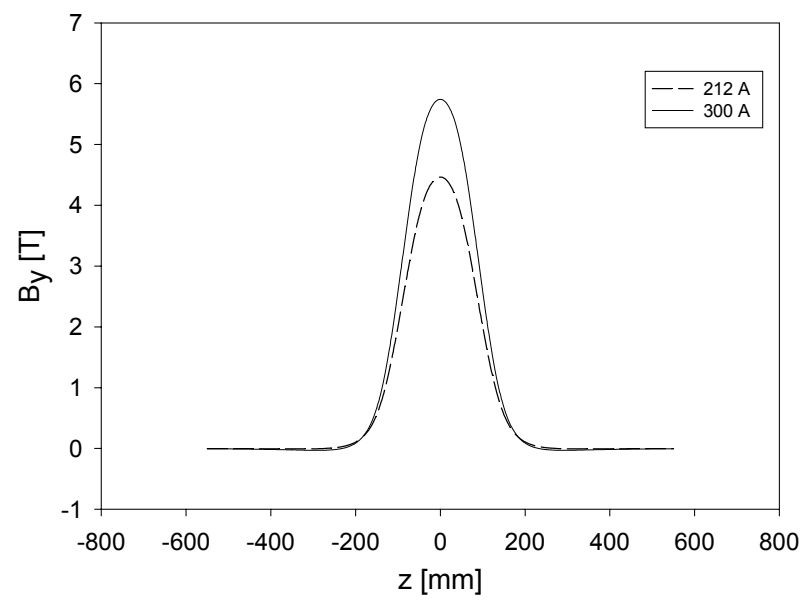

Fig. 3 Axial field profile of Superbend magnet \#3, at 212 A and $300 \mathrm{~A}$.

\section{CRYOGENIC PERFORMANCE}

In order to assess the cryogenic/operational state of the Superbends, each magnet is equipped with 8 CERNOX temperature sensors purchased from Lakeshore Cryotronics. Table V presents the measured Superbend temperatures for the case of refrigeration provided by the cryocooler, Sumitomo model SRDK-415, with the magnet operating at $300 \mathrm{~A}$.

TABLE V

MEASURED TEMPERATURES AT I $=300$ A FOR THE SUPERBENDS

\begin{tabular}{ccccc}
\hline \hline $\begin{array}{c}\text { Temperature } \\
\text { Location }\end{array}$ & $\begin{array}{c}\# \mathbf{1} \\
{[\mathbf{K}]}\end{array}$ & $\begin{array}{c}\mathbf{\# 2} \\
{[\mathbf{K}]}\end{array}$ & $\begin{array}{c}\mathbf{\# 3} \\
{[\mathbf{K}]}\end{array}$ & $\begin{array}{c}\mathbf{\# 4} \\
{[\mathbf{K}]}\end{array}$ \\
\hline Stage 1 & 38.8 & 44.4 & 43.0 & 45.2 \\
Stage 2 & 4.05 & 3.56 & 3.75 & 3.92 \\
HTS Lead A & 56.5 & 58.1 & 57.0 & 57.4 \\
HTS Lead B & 56.5 & 59.1 & 57.0 & 57.0 \\
Upper Coil & 4.37 & 3.98 & 4.27 & 4.33 \\
Lower Coil & 4.41 & 4.01 & 4.28 & 4.36 \\
Upper Yoke & 4.55 & 4.17 & 4.36 & 4.48 \\
Lower Yoke & 4.60 & 4.21 & 4.38 & 4.54 \\
\hline \hline
\end{tabular}

The temperatures in Table $\mathrm{V}$ lie close to the expected values and demonstrate that the cryogenic design goals are met. No cryogenic fluids are consumed as long as the cryocooler is operational.

An important operational parameter is the rate at which the magnets can be ramped from the injection current of $212 \mathrm{~A}$, corresponding to $1.5 \mathrm{GeV}$ beam energy, to $298 \mathrm{~A}$, corresponding to the $1.9 \mathrm{GeV}$ nominal operating point. Tests demonstrate that the magnets can be reliably ramped at 1 $\mathrm{A} / \mathrm{sec}$ when operating with the cryocooler. The limitation is due to eddy current heating in the conductor. Prior to installation the Superbends were cycled a minimum of 1000 times between the above current limits at $0.8 \mathrm{~A} / \mathrm{sec}$ to establish confidence in reliable operation.

In the event of cryocooler failure, the system is operated using cryogenic fluids provided by external transfer from $\mathrm{LN}_{2}$ and LHe dewars located outside the ALS storage ring to vessels inside the cryostat. When operating in this mode, the internal vessels are fitted with relief valves having a 1.035 bar cracking pressure. Liquid nitrogen provides cooling for the warm end of the HTS current leads. In addition it provides thermal shielding for the superconducting coils and the cold iron yoke from heat conducted through the leads and cryocooler. For Magnet \#2 we find that if the $\mathrm{LN}_{2}$ level is maintained between 95 and 30 per cent the temperature of the warm end of the HTS lead remains at $84 \mathrm{~K}$ or less, which is within the specification of the HTS lead. The 35 liter $\mathrm{LN}_{2}$ vessel must be refilled about every 14 hours to maintain this level. Liquid helium cools the superconducting coils and the 1.5 Tonne iron yoke by means of copper conduction paths. For Magnet \#2 the temperature of the coils is maintained at $4.8 \mathrm{~K}$ or less when the helium level is greater than 50 per cent. This requires that we transfer liquid helium about every five hours.

For maintenance purposes the Superbend cryostat has a port that is used to gain access to internal components. The cold mass is equipped with tubes for rapid cooldown and electrical heaters for rapid warmup. The cryocooler must be replaced every 10,000 hours with one that has been factoryreconditioned, so this was positioned to allow rapid changeout. We find that we need about 30 hours to warm from operating temperature to $300 \mathrm{~K}, 4$ hours to change cryocoolers, and about 30 hours to pump down and cool from $300 \mathrm{~K}$ to operating temperature.

\section{ALS COMMISSIONING}

The installation of the Superbends and the conversion of the ALS lattice was accomplished on schedule within a 10 day period. Currently the storage ring is being recommissioned. Early results are very encouraging. Within one day of the start of commissioning electrons were stored and ramped to $1.9 \mathrm{GeV}$. This was possible due to the small orbit distortions and accurate ramping table predictions confirming the accuracy of the magnetic measurements. Within a week the storage ring was operated at full current and full energy. Currently the commissioning team is continuing to evaluate the performance of the storage ring with Superbends.

\section{REFERENCES}

[1] C. E. Taylor and S. Caspi, "A 6.3 T Bend Magnet for the Advanced Light Source," IEEE Trans. Magnetics 32, No. 4, 1996, pp. 2175-2178.

[2] C. E. Taylor, et al., "Test of a High-Field Magnet for the ALS," Transactions Applied Superconductivity 9, No. 2, 1999, pp. 479-482.

[3] J. Zbasnik, et al., "ALS Superbend Magnet System," IEEE Trans. on Applied Superconductivity, Vol. 11, No. 1, March, 2001, pp. 25312534.

[4] Advanced Light Source Division, "Superbend Magnet System Conceptual Design Report," Lawrence Berkeley National Laboratory Publication, PUB-5457, Apr. 2000.

[5] D. Robin, et al., "Superbend Project at The Advanced Light Source", Proceedings of the 2001 Particle Accelerator Conference, (2001).

[6] B. Berkes, "Hall Generators,", CERN Acc. School (1992). 